\title{
Testosterone and Its Bioactive Components Are Associated with Libido and the Metabolic Syndrome in Men
}

\author{
H. Alidu ${ }^{*}$, N. Amidu², W. K. B. A. Owiredu ${ }^{3}$, C. K. Gyasi-Sarpong4, A. T. Bawah'1, P. P. M. Dapare ${ }^{2}$, \\ E. B. Agyemang Prempeh ${ }^{5}$
}

\begin{abstract}
${ }^{1}$ Department of Medical Laboratory Science, University of Health and Allied Sciences, Ho, Ghana
${ }^{2}$ Department of Biomedical Laboratory Science, University for Development Studies, Tamale, Ghana

${ }^{3}$ Department of Molecular Medicine, Kwame Nkrumah University of Science \& Technology, Kumasi, Ghana

${ }^{4}$ Department of Surgery (Urology), School of Medical Sciences/Komfo Anokye Teaching Hospital, Kumasi, Ghana

${ }^{5}$ Department of Academic Affairs, University of Health and Allied Sciences, Ho, Ghana

Email: *wiisibie@yahoo.com, nafamidu@yahoo.com, wkbaowiredu@yahoo.com, gaysek@yahoo.com, ahmed024gh@yahoo.com,

peterpauldapare@yahoo.com, eprempeh@uhas.edu.gh
\end{abstract}

How to cite this paper: Alidu, H., Amidu, N., Owiredu, W.K.B.A., Gyasi-Sarpong, C.K., Bawah, A.T., Dapare, P.P.M. and Agyemang Prempeh, E.B. (2017) Testosterone and Its Bioactive Components Are Associated with Libido and the Metabolic Syndrome in Men. Advances in Sexual Medicine, 7, 105-119.

https://doi.org/10.4236/asm.2017.72008

Received: March 24, 2017

Accepted: April 27, 2017

Published: April 30, 2017

Copyright $\odot 2017$ by authors and Scientific Research Publishing Inc. This work is licensed under the Creative Commons Attribution International License (CC BY 4.0).

http://creativecommons.org/licenses/by/4.0/

(c) (i) Open Access

\begin{abstract}
Background: Some evidence has shown that the prevalence of hypoactive sexual desire and erectile dysfunction (ED) is associated with testosterone levels whilst higher levels of testosterone have been reported to increase the frequency of intercourse. Available evidence points towards an etiologic role for hypogonadism in the causation of diabetes, metabolic syndrome (Mets) and sexual dysfunction (SD) even though the exact pathophysiological linkage is yet to be fully elucidated. This study therefore sought to evaluate the impact of testosterone as well as its bioactive components on both the MetS and SD. Method: Diabetic men engaged in a stable heterosexual relationship for at least 2 years were recruited for this study. Participants were at least 18 years and provided an informed and signed consent to partake in this study. Fasting blood samples were taken from the participants for biochemical and hormonal assay. The participants were then evaluated using the Golombok Rust Inventory of Sexual Satisfaction for males (GRISS-M). Metabolic syndrome was assessed using the NCEP-ATP III, IDF and WHO criteria. All data analyses were performed using the SPSS software, version 11.0 systat, Inc. Germany and GraphPad Prism, version 5.0, San Diego California, USA. Results: The mean total, free and bioavailable testosterone as well as SHBG recorded among the participants was $7.10 \pm 1.23 \mathrm{ng} / \mathrm{ml}, 0.10 \pm 0.01 \mathrm{ng} / \mathrm{ml}, 7.01 \pm 1.41 \mathrm{ng} / \mathrm{ml}$ and $4.33 \pm 1.12 \mathrm{nmol} / \mathrm{l}$ respectively. Subjects with the MetS showed significantly lower SHBG levels in comparison with subjects without the MetS. Participants with raised triglyceride levels showed significantly lower levels of total, free and bioavailable testosterone when compared to participant without
\end{abstract}


raised triglycerides. Participants with problems of avoidance and infrequency of sexual activity showed significantly lower levels of total, free and bioavailable testosterone when compared to participants without avoidance or infrequency problems. The $+\mathrm{SD} /+$ MetS group recorded the lowest SHBG levels. Conclusion: Testosterone and its bioactive components are associated with male libido as well as the metabolic syndrome. Low levels of free and bioavailable testosterone are true determinants of the MetS in men.

\section{Keywords}

Hypogonadism, Sexual Dysfunction, Metabolic Syndrome, Type II Diabetes, Testosterone

\section{Background}

Sexuality is a complex process and a multidimensional phenomenon that incorporates biological, psychological, interpersonal and behavioral dimensions [1] [2]. Libido is defined as the biological need for sexual activity and drives men towards a need for sexual intimacy. The intensity of this drive may vary between individuals as well as within the same individual over a given period of time. Some evidence has linked testosterone levels to increased intensity of this need for sexual activity [3] in healthy older but not younger men [2]. Testosterone is taught to be involved in promoting sexual activity and this appears to be mediated by an increase in dopamine release possibly by up regulation of NO synthesis. Higher levels of testosterone in individuals have been shown to shorten the latency of erection that is stimulated by erotic images and some evidence has shown that the replacement of testosterone in hypogonadal men restores sexual interest [4] whilst its withdrawal results in a decreased libido [5].

Recently emerged evidence has suggested that the secretion or activity of NO an important vasodilator necessary for erection to occur is modulated by gonadal androgens [6] with some researchers proposing a possible role for the involvement of peripheral androgens [7]. Androgens have been shown to improve Nocturnal Penile Tumescence, (NPT) [6] [7] and this has led some researchers proposing that sexual behaviors as well as penile erection are dependent on androgen levels with androgens potentially acting both centrally and peripherally [8], with the possible presence of both androgen sensitive and androgen insensitive central pathways controlling androgen action [6].

Erectile dysfunction (ED; or impotence) and premature ejaculation (PE) are the two most prevalent complaints in male sexual dysfunction (SD) [9]. The worldwide incidence of ED is estimated at over 152 million men [9] [10] with an estimated projection of about 322 men worldwide developing ED by the year 2025.

Disorders of desire or decreased libido are characterized by a lack or absence of desire for sexual activity or the absence of sexual fantasies [10]. The absence of this fundamental requirement in human sexual activity could impact on a 
man's sexual expressions, cause dissatisfaction for sexual activity and compromise one's sexual quality of life.

There is no universal agreement regarding the definition of hypogonadism. However it is generally accepted that hypogonadism is used to refer to the presence of persistently low circulating testosterone levels relative to the normal population [11]. Low testosterone and sex hormone binding globulin (SHBG) levels have also been reported as risk factors for the development of the MetS. A higher prevalence of MetS in men treated with Androgen Deprivation Therapy (ADT) reported by some researchers [12] provided evidence of a strong linkage of hypogonadism in the eventual development of the MetS. The role of hypogonadism in the accumulation of body fat mass is quite evidenced from these studies and the extent of this relationship was demonstrated when Wang and his colleagues [13] showed that the relationship between androgens and body fat mass was even dose dependent.

Testosterone is largely bound to sex hormone binding globulin (SHBG) and this could influence the availability of free testosterone levels in the body. The decreasing levels of SHBG with age [11] could influence the fractions of free and bioavailable testosterone levels in men thus impacting the development of the MetS.

Androgens have been reported to promote myogenesis and inhibit adipogenesis [14], thus androgen levels are likely to influence the development of the MetS in both sexes. The finding that testosterone levels also modulate NO availability and activity [15] [16] and possibly endothelial function [17] [18] provides strong reasons why hypogonadism is likely to influence both the MetS and SD. Some researchers have reported [19] a significant increase in fat free mass and a significant decrease in body fat mass with testosterone therapy. Other researchers, investigating the impact of treatment regimens on sexual dysfunction and symptoms of the metabolic syndrome, reported that improvements in androgen-related functions were more favorable when plasma testosterone levels were in the mid-normal range [20]. Some evidence has shown that the prevalence of hypoactive sexual desire and ED is associated with testosterone levels whilst higher levels of testosterone increased the frequency of intercourse [21]. Available evidence points towards an etiologic role for hypogonadism in the causation of diabetes, Mets and SD even though the exact pathophysiological linkage is yet to be fully elucidated. Wang and his colleagues earlier provided some evidence of an association between Testosterone levels and the MetS as well as sexual function [22]. The exact component of testosterone which correlates better with true hypogonadism and subsequently impacts on sexual function and the MetS is also still an issue of debate. This study therefore sought to evaluate the components of testosterone and their impact on both the MetS and SD and its domains.

\section{Methods}

Participants in this study were 130 type II diabetic males who attend the diabetic clinic at the Maamobi General Hospital in Accra and were being managed on 
oral diabetic medications. Participants were at least 18 years and above and were consecutively sampled if they were actively engaged in a stable heterosexual relationship which was defined as having engaged in a continuous heterosexual relationship for a minimum of two years before enrollment in this study. Each participant was required to sign an informed consent to participate in the study. Patients who were not known type II diabetics or had obvious physical disabilities were excluded from the study. Ethical approval was obtained from the Committee on Human Research Publication and Ethics (CHRPE) of the School of Medical Science and the Komfo Anokye Teaching Hospital (KATH), Kumasi. Sexual function of the participants was evaluated using the Golombok Rust Inventory of Sexual Satisfaction for males (GRISS-M) with participants grouped as having Sexual Dysfunction (SD) when their stanine scores was equal to or greater than five (5). Subscales of Impotence (IMP), Premature Ejaculation (PE), Non-sensuality (NS) Avoidance (AV), Dissatisfaction (DIS), Non-communication (NC) and Infrequency (INF) was also computed using their stanine scores. The NCEPATP III [23], IDF [24] and WHO criteria of the MetS were used to assess the metabolic syndrome among all the participants in the study. Ten (10) mls of venous blood was taken from each participants in the morning hours between 06.00 to $09.00 \mathrm{GMT}$ in the fasting state, five (5) mls was put into a fluoride oxalate tube which was well mixed and centrifuged at $3000 \mathrm{~g}$ for 5 minutes. The other 5 (mls) was put in an evacuated gel tube (Becton Dickinson, Rutherford, $\mathrm{NJ}$ ) and allowed enough time to clot and then centrifuged for 5 minutes at 3000 $\mathrm{g}$ and the serum separated into a plain tube. Samples in the fluoride oxalate tubes were used for fasting blood glucose measurement whilst the serum obtained in the plain tube was stored at a temperature of $-80^{\circ} \mathrm{C}$ until time for biochemical and hormonal assay. SHBG was measured using the Sandwich ELISA method whilst the competitive-ELISA method was used to assay the testosterone levels. Lipid profile and fasting blood glucose levels were determined using the BT 5000 Random Access Chemistry Analyzer (Biotecnica, Italy) and the JAS Diagnostics ${ }^{\otimes}$ reagent kits were used in all of these assays. The hormonal assays were done using the Elabscience ${ }^{\circledast}$ reagent kits on the AxSYM analyzer. The methods adopted by the automated instruments for the determination of biochemical and hormonal parameters were done according to the reagent manufacturers' instructions (JAS Diagnostics, Inc. Miami Florida, USA and Elabscience Biotechnology Co. Ltd., Hubei Province, China).

\section{Statistical Analysis}

All data analyses were performed using the SPSS software, version 11.0 systat, Inc. Germany and Graph Pad Prism, version 5.0, San Diego California, USA. Data presentation was done as the mean $\pm S D$ or percentages. In all the statistical analysis, a value of $\mathrm{p}<0.05$ was considered to be significant.

\section{Results}

The mean total testosterone, free testosterone, bioavailable testosterone and 
SHBG recorded among the participants was $7.10 \pm 1.23 \mathrm{ng} / \mathrm{ml}, 0.10 \pm 0.01 \mathrm{ng} /$ $\mathrm{ml}, 7.01 \pm 1.41 \mathrm{ng} / \mathrm{ml}$ and $4.33 \pm 1.12 \mathrm{nmol} / \mathrm{l}$ respectively. When the study participants were stratified by the presence or absence of the MetS, participants with the MetS recorded significantly lower levels of total testosterone for all criteria of the MetS. Participants with the MetS also recorded significantly lower levels of free (IDF) and bioavailable testosterone (IDF). Furthermore, participants with the MetS also recorded lower levels of SHBG in comparison with those without the MetS (ATP III and WHO) (Table 1).

There was no significant difference observed in the total testosterone, free testosterone, and bioavailable testosterone levels of participants when participants with 1,2, 3 and 4 component score of the MetS were compared with regards to the IDF and WHO criteria. However the ATP III criteria recorded significant differences in the levels of total and bioavailable testosterone with decreasing levels of total and bioavailable testosterone along the 1, 2, 3 and 4 MetS score groups. Participants with four components of the MetS recorded hypogonadal levels of total and bioavailable testosterone. There was however no differences in the free testosterone and SHBG levels when the same comparism was done for the 1, 2, 3 and 4 component score groups (Table 2).

When participants were stratified based on the presence or absence of the various specific MetS components, participants with Abdominal Obesity showed no significant difference in any of the assayed parameters whilst participants with Central Obesity showed significantly lower levels of SHBG as compared to participants without Central Obesity. Participants with raised FBG showed no significant difference in their total, free and bioavailable testosterone levels as

Table 1. Testosterone levels Stratified by Presence or Absence of MetS.

\begin{tabular}{|c|c|c|c|c|}
\hline Hormonal Variable & TOTAL & No MetS & MetS & $P$ Value \\
\hline \multicolumn{5}{|c|}{ ATP III } \\
\hline Total Testosterone (ng/ml) & $7.10 \pm 1.23$ & $9.07 \pm 2.43$ & $4.43 \pm 1.42$ & 0.0410 \\
\hline Free Testosterone (ng/ml) & $0.10 \pm 0.01$ & $1.01 \pm 0.65$ & $0.06 \pm 0.00$ & 0.1464 \\
\hline Bioavailable Testosterone (ng/ml) & $7.01 \pm 1.41$ & $9.06 \pm 3.42$ & $4.36 \pm 1.52$ & 0.1002 \\
\hline SHBG (nmol/l) & $4.33 \pm 1.12$ & $5.34 \pm 1.61$ & $3.52 \pm 1.03$ & 0.02145 \\
\hline \multicolumn{5}{|c|}{ IDF } \\
\hline Total Testosterone (ng/ml) & & $9.27 \pm 2.75$ & $4.51 \pm 1.64$ & 0.0339 \\
\hline Free Testosterone (ng/ml) & & $1.17 \pm 0.54$ & $0.24 \pm 0.05$ & 0.0394 \\
\hline Bioavailable Testosterone (ng/ml) & & $8.08 \pm 1.09$ & $4.30 \pm 1.44$ & 0.0483 \\
\hline SHBG (nmol/l) & & $5.62 \pm 2.43$ & $3.89 \pm 0.97$ & 0.1087 \\
\hline \multicolumn{5}{|c|}{ WHO } \\
\hline Total Testosterone (ng/ml) & & $10.83 \pm 3.71$ & $2.21 \pm 0.84$ & 0.0392 \\
\hline Free Testosterone (ng/ml) & & $1.07 \pm 0.50$ & $0.22 \pm 0.03$ & 0.2946 \\
\hline Bioavailable Testosterone (ng/ml) & & $10.08 \pm 4.66$ & $2.01 \pm 0.68$ & 0.6201 \\
\hline SHBG (nmol/l) & & $5.26 \pm 1.03$ & $3.99 \pm 1.11$ & 0.0277 \\
\hline
\end{tabular}

No MetS-With no metabolic syndrome/MetS = with metabolic syndrome. 
Table 2. Testosterone levels stratified by MetS component scores.

\begin{tabular}{|c|c|c|c|c|c|c|}
\hline \multirow{2}{*}{ Hormonal variables } & \multicolumn{4}{|c|}{ MetS Score } & \multirow{2}{*}{ F Value } & \multirow{2}{*}{$\begin{array}{c}\mathbf{P} \\
\text { Value }\end{array}$} \\
\hline & 1 & 2 & 3 & 4 & & \\
\hline \multicolumn{7}{|c|}{ ATP III } \\
\hline Total Testosterone (ng/ml) & $10.54 \pm 2.61$ & $7.46 \pm 1.83$ & $5.21 \pm 1.24$ & $2.17 \pm 0.46$ & $\mathrm{~F}_{3,122}=1.2755$ & 0.0434 \\
\hline Free Testosterone (ng/ml) & $1.66 \pm 0.53$ & $1.09 \pm 0.64$ & $1.04 \pm 0.29$ & $1.02 \pm 0.04$ & $\mathrm{~F}_{3,122}=0.7389$ & 0.1235 \\
\hline Bioavailable Testosterone (ng/ml) & $8.41 \pm 2.26$ & $6.33 \pm 1.39$ & $4.13 \pm 1.01$ & $1.14 \pm 0.28$ & $\mathrm{~F}_{3,122}=1.1590$ & 0.0432 \\
\hline SHBG (nmol/l) & $4.59 \pm 1.86$ & $3.64 \pm 1.04$ & $4.22 \pm 1.61$ & $3.45 \pm 1.08$ & $\mathrm{~F}_{3,122}=1.1194$ & 0.1522 \\
\hline \multicolumn{7}{|c|}{ IDF } \\
\hline Total Testosterone (ng/ml) & $5.52 \pm 1.43$ & $4.95 \pm 0.37$ & $4.43 \pm 1.70$ & $2.07 \pm 0.89$ & $\mathrm{~F}_{3,122}=2.0050$ & 0.1227 \\
\hline Free Testosterone (ng/ml) & $0.18 \pm 0.09$ & $0.10 \pm 0.01$ & $0.20 \pm 0.08$ & $0.09 \pm 0.01$ & $\mathrm{~F}_{3,122}=1.9280$ & 0.1345 \\
\hline Bioavailable Testosterone (ng/ml) & $5.36 \pm 1.41$ & $4.82 \pm 1.33$ & $4.22 \pm 1.29$ & $2.00 \pm 0.94$ & $\mathrm{~F}_{3,122}=1.9563$ & 0.1291 \\
\hline SHBG (nmol/l) & $5.15 \pm 1.75$ & $4.25 \pm 1.41$ & $4.76 \pm 1.95$ & $4.00 \pm 1.86$ & $\mathrm{~F}_{3,122}=0.1228$ & 0.8562 \\
\hline \multicolumn{7}{|c|}{ WHO } \\
\hline Total Testosterone (ng/ml) & $4.52 \pm 1.43$ & $4.30 \pm 0.35$ & $3.21 \pm 1.02$ & $2.30 \pm 1.98$ & $F_{3,122}=0.2893$ & 0.3854 \\
\hline Free Testosterone (ng/ml) & $0.18 \pm 0.06$ & $0.01 \pm 0.00$ & $0.14 \pm 0.03$ & $0.12 \pm 0.08$ & $\mathrm{~F}_{3,122}=0.9876$ & 0.4833 \\
\hline Bioavailable Testosterone (ng/ml) & $4.36 \pm 1.41$ & $4.25 \pm 1.28$ & $3.08 \pm 1.06$ & $2.15 \pm 0.87$ & $\mathrm{~F}_{3,122}=0.3852$ & 0.3965 \\
\hline SHBG (nmol/l) & $5.15 \pm 1.74$ & $4.68 \pm 1.52$ & $4.26 \pm 1.30$ & $4.07 \pm 1.54$ & $\mathrm{~F}_{3,122}=0.1119$ & 0.7492 \\
\hline
\end{tabular}

well as SHBG levels in comparison with participants without raised FBG. Participants with raised Triglyceride levels showed significantly lower levels of total, free and bioavailable testosterone when compared to participant without raised Triglyceride levels. There was however no observed differences in the total testosterone, free testosterone, bioavailable testosterone and SHBG levels when participants with or without raised BP, reduced HDL-cholesterol and Dyslipidemia were compared, irrespective of the MetS criteria used (Table 3 and Table 4).

When the diabetic male participants were stratified based on the presence or absence of SD sub scales, participants who had impotence showed no difference in their total testosterone, free testosterone, bioavailable testosterone and SHBG levels in comparison with participants without difficulties of impotence. Participants with difficulties of premature ejaculation, non-sensuality, dissatisfaction and non-communication also showed no difference in their levels of total, free and bioavailable testosterone as well as SHBG levels in comparison with participants without these difficulties. Participants with difficulties of avoidance and infrequency in sexual activity showed significantly lower hypogonadal levels of total, free and bioavailable testosterone when compared to participants without avoidance problems. They however showed no difference in their SHBG levels (Table 5).

When the different shades of the SD/MetS groups were compared for their total, bioavailable and free testosterone levels, there was no difference recorded amongst these groups. However, when the same groups were compared for their SHBG levels, significantly decreasing levels of SHBG $\left(\mathrm{p}=0.0205, \mathrm{~F}_{3,122}=1.148\right)$ were recorded (ATP III), with SHBG levels being highest among the -SD/-MetS 
Table 3. Total and Free Testosterone levels stratified by absence and presence of MetS Components.

\begin{tabular}{|c|c|c|c|c|c|c|}
\hline \multirow[b]{2}{*}{ Variable } & \multicolumn{3}{|c|}{ Total Testosterone(ng/ml) } & \multicolumn{3}{|c|}{ Free Testosterone(ng/ml) } \\
\hline & Absent & Present & P Value & Absent & Present & $P$ Value \\
\hline \multicolumn{7}{|c|}{ ATP III } \\
\hline Abdominal Obesity-WC & $16.75 \pm 3.76$ & $7.88 \pm 1.34$ & 0.2339 & $1.87 \pm 0.64$ & $1.01 \pm 0.09$ & 0.2273 \\
\hline Raised FBG & $11.63 \pm 2.21$ & $6.66 \pm 1.34$ & 0.3499 & $1.04 \pm 0.00$ & $0.92 \pm 0.08$ & 0.5382 \\
\hline Raised TG & $15.24 \pm 3.93$ & $13.61 \pm 4.37$ & 0.0115 & $2.33 \pm 0.08$ & $1.76 \pm 0.93$ & 0.0485 \\
\hline Raised BP & $11.26 \pm 2.11$ & $7.49 \pm 1.96$ & 0.5732 & $1.05 \pm 0.49$ & $0.56 \pm 0.07$ & 0.5894 \\
\hline Reduced HDL-C & $8.38 \pm 2.27$ & $5.30 \pm 1.32$ & 0.5812 & $0.72 \pm 0.05$ & $0.21 \pm 0.06$ & 0.4968 \\
\hline \multicolumn{7}{|c|}{ IDF } \\
\hline Abdominal Obesity-WC & $10.54 \pm 2.33$ & $6.54 \pm 1.32$ & 0.2022 & $0.54 \pm 0.00$ & $0.34 \pm 0.11$ & 0.3444 \\
\hline Raised FBG & $11.35 \pm 2.47$ & $6.36 \pm 1.82$ & 0.3499 & $0.69 \pm 0.06$ & $0.21 \pm 0.06$ & 0.5382 \\
\hline Raised TG & $15.07 \pm 3.22$ & $11.65 \pm 3.43$ & 0.0115 & $1.86 \pm 0.08$ & $1.32 \pm 0.31$ & 0.0285 \\
\hline Raised BP & $5.92 \pm 1.31$ & $7.22 \pm 2.25$ & 0.7216 & $1.03 \pm 0.26$ & $0.28 \pm 0.06$ & 0.7305 \\
\hline Reduced HDL-C & $8.48 \pm 1.99$ & $4.32 \pm 1.14$ & 0.4087 & $0.34 \pm 0.08$ & $0.15 \pm 0.04$ & 0.3499 \\
\hline \multicolumn{7}{|c|}{ WHO } \\
\hline Central Obesity-WHR & $9.88 \pm 2.53$ & $5.21 \pm 1.10$ & 0.4332 & $0.63 \pm 0.09$ & $0.40 \pm 0.05$ & 0.5098 \\
\hline Raised FBG & $11.62 \pm 4.37$ & $6.82 \pm 2.04$ & 0.3499 & $1.28 \pm 0.93$ & $0.58 \pm 0.13$ & 0.5382 \\
\hline Dyslipidemia & $6.44 \pm 1.34$ & $4.64 \pm 1.66$ & 0.5863 & $1.22 \pm 0.27$ & $0.43 \pm 0.03$ & 0.9208 \\
\hline Raised BP & $9.47 \pm 1.84$ & $6.44 \pm 2.48$ & 0.5578 & $1.82 \pm 0.76$ & $0.85 \pm 0.19$ & 0.4422 \\
\hline
\end{tabular}

Table 4. Bioavailable Testosterone and SHBG stratified by presence or absence of MetS Component.

\begin{tabular}{|c|c|c|c|c|c|c|}
\hline \multirow[b]{2}{*}{ Variable } & \multicolumn{3}{|c|}{ Bioavailable Testosterone(ng/ml) } & \multicolumn{3}{|c|}{ SHBG (nmol/l) } \\
\hline & Absent & Present & P Value & Absent & Present & P Value \\
\hline \multicolumn{7}{|c|}{ ATP III } \\
\hline Abdominal Obesity-WC & $13.44 \pm 3.12$ & $6.65 \pm 1.32$ & 0.2151 & $3.58 \pm 1.07$ & $3.10 \pm 1.06$ & 0.6493 \\
\hline Raised FBG & $10.32 \pm 2.04$ & $6.73 \pm 1.51$ & 0.3865 & $3.87 \pm 1.05$ & $3.15 \pm 1.02$ & 0.3328 \\
\hline Raised TG & $15.45 \pm 3.22$ & $9.88 \pm 2.10$ & 0.0033 & $3.16 \pm 1.19$ & $2.29 \pm 0.62$ & 0.0124 \\
\hline Raised BP & $11.38 \pm 3.36$ & $6.50 \pm 2.39$ & 0.5395 & $3.45 \pm 1.02$ & $4.63 \pm 1.27$ & 0.0762 \\
\hline Reduced HDL-C & $7.48 \pm 1.33$ & $4.53 \pm 1.86$ & 0.5322 & $3.57 \pm 1.06$ & $3.68 \pm 1.06$ & 0.3852 \\
\hline \multicolumn{7}{|c|}{ IDF } \\
\hline Abdominal Obesity-WC & $10.34 \pm 2.43$ & $5.45 \pm 1.33$ & 0.4642 & $4.22 \pm 1.47$ & $3.54 \pm 1.08$ & 0.1433 \\
\hline Raised FBG & $10.25 \pm 2.38$ & $6.62 \pm 1.34$ & 0.3865 & $3.78 \pm 1.37$ & $3.47 \pm 1.43$ & 0.3562 \\
\hline Raised TG & $15.31 \pm 4.33$ & $10.38 \pm 2.19$ & 0.0039 & $3.25 \pm 1.09$ & $2.54 \pm 0.63$ & 0.0024 \\
\hline Raised BP & $5.31 \pm 1.66$ & $7.29 \pm 2.45$ & 0.7513 & $3.98 \pm 1.01$ & $3.87 \pm 1.03$ & 0.3591 \\
\hline Reduced HDL-C & $8.30 \pm 2.39$ & $4.36 \pm 1.48$ & 0.3702 & $3.86 \pm 1.07$ & $3.78 \pm 1.06$ & 0.6802 \\
\hline \multicolumn{7}{|c|}{ WHO } \\
\hline Central Obesity-WHR & $8.36 \pm 2.32$ & $5.54 \pm 1.75$ & 0.4370 & $5.23 \pm 1.47$ & $4.44 \pm 1.34$ & 0.0432 \\
\hline Raised FBG & $10.37 \pm 3.53$ & $6.48 \pm 1.33$ & 0.3865 & $4.15 \pm 1.52$ & $3.98 \pm 1.02$ & 0.5722 \\
\hline Dyslipidemia & $8.52 \pm 2.33$ & $6.56 \pm 1.54$ & 0.7121 & $3.63 \pm 1.16$ & $2.00 \pm 0.43$ & 0.1739 \\
\hline Raised BP & $9.35 \pm 2.73$ & $8.78 \pm 1.43$ & 0.4985 & $4.63 \pm 1.58$ & $5.25 \pm 1.44$ & 0.2634 \\
\hline
\end{tabular}


group $(6.46 \pm 1.30)$, followed by the $+\mathrm{SD} /-\mathrm{MetS}$ group $(5.16 \pm 1.45)$ and the $-\mathrm{SD} /+$ MetS group $(3.92 \pm 1.08)$. The $+\mathrm{SD} /+\mathrm{MetS}$ group recorded the lowest SHBG $(3.47 \pm 1.01)$ levels (Table 6).

Table 5. Total, Free, Bioavailable Testosterone and SHBG levels stratified by absence or presence of domains of SD.

\begin{tabular}{|c|c|c|c|c|c|c|}
\hline \multirow{2}{*}{ Variable } & \multicolumn{3}{|c|}{ Total Testosterone(ng/ml) } & \multicolumn{3}{|c|}{ Free Testosterone(ng/ml) } \\
\hline & Absent & Present & $P$ Value & Absent & Present & P Value \\
\hline Impotence & $8.37 \pm 2.29$ & $7.58 \pm 2.34$ & 0.7825 & $0.79 \pm 0.10$ & $0.53 \pm 0.08$ & 0.5978 \\
\hline Premature ejaculation & $5.69 \pm 2.56$ & $9.52 \pm 2.38$ & 0.4240 & $0.31 \pm 0.09$ & $0.50 \pm 0.13$ & 0.4873 \\
\hline Avoidance & $11.37 \pm 4.62$ & $2.12 \pm 0.33$ & 0.0416 & $0.52 \pm 0.15$ & $0.12 \pm 0.09$ & 0.0349 \\
\hline Dissatisfaction & $8.49 \pm 2.62$ & $7.36 \pm 2.60$ & 0.8614 & $0.38 \pm 0.12$ & $0.18 \pm 0.06$ & 0.6957 \\
\hline Non communication & $5.82 \pm 1.29$ & $8.45 \pm 2.22$ & 0.6454 & $0.41 \pm 0.14$ & $0.38 \pm 0.11$ & 0.9352 \\
\hline \multirow[t]{3}{*}{ Infrequency } & $5.43 \pm 1.54$ & $2.05 \pm 0.15$ & 0.0445 & $0.69 \pm 0.08$ & $0.43 \pm 0.17$ & 0.0481 \\
\hline & \multicolumn{3}{|c|}{ Bioavailable Testosterone (ng/ml) } & \multicolumn{3}{|c|}{ SHBG (nmol/1) } \\
\hline & Absent & Present & $P$ Value & Absent & Present & P Value \\
\hline Impotence & $7.52 \pm 2.33$ & $6.92 \pm 2.02$ & 0.7441 & $5.01 \pm 2.02$ & $3.11 \pm 1.93$ & 0.1396 \\
\hline Premature ejaculation & $5.36 \pm 1.62$ & $8.90 \pm 1.33$ & 0.4064 & $3.91 \pm 1.98$ & $5.03 \pm 2.43$ & 0.1734 \\
\hline Non sensuality & $7.38 \pm 1.97$ & $7.04 \pm 2.02$ & 0.8995 & $3.62 \pm 1.87$ & $4.02 \pm 2.66$ & 0.2964 \\
\hline Avoidance & $10.81 \pm 3.55$ & $2.02 \pm 0.09$ & 0.0463 & $5.01 \pm 2.23$ & $3.02 \pm 1.91$ & 0.1836 \\
\hline Dissatisfaction & $7.94 \pm 1.38$ & $7.16 \pm 2.52$ & 0.9001 & $3.95 \pm 1.71$ & $4.03 \pm 2.88$ & 0.2841 \\
\hline Non communication & $5.41 \pm 1.24$ & $7.44 \pm 1.67$ & 0.6802 & $3.09 \pm 1.87$ & $4.19 \pm 2.48$ & 0.1843 \\
\hline Infrequency & $3.36 \pm 0.37$ & $1.36 \pm 0.76$ & 0.0433 & $4.01 \pm 2.21$ & $4.66 \pm 2.14$ & 0.4398 \\
\hline
\end{tabular}

Table 6. Total, Free and Bioavailable Testosterone levels stratified by shades of SD/MetS.

\begin{tabular}{|c|c|c|c|c|c|c|}
\hline Hormonal Variables & $-\mathrm{SD} /-\mathrm{MetS}$ & $+\mathrm{SD} /-\mathrm{MetS}$ & $-\mathrm{SD} /+\mathrm{MetS}$ & $+\mathrm{SD} /+\mathrm{MetS}$ & F Value & P Value \\
\hline \multicolumn{7}{|c|}{ ATP III } \\
\hline Total Testosterone (ng/ml) & $7.26 \pm 2.02$ & $6.74 \pm 1.01$ & $5.89 \pm 1.40$ & $3.79 \pm 1.07$ & $\mathrm{~F}_{3,122}=1.4070$ & 0.2494 \\
\hline Free Testosterone (ng/ml) & $1.01 \pm 0.09$ & $0.60 \pm 0.00$ & $0.40 \pm 0.00$ & $0.10 \pm 0.00$ & $\mathrm{~F}_{3,122}=1.0760$ & 0.3660 \\
\hline Bioavailable Testosterone (ng/ml) & $6.23 \pm 1.48$ & $6.18 \pm 1.09$ & $5.69 \pm 1.73$ & $3.65 \pm 0.91$ & $\mathrm{~F}_{3,122}=1.3040$ & 0.2814 \\
\hline SHBG (nmol/l) & $6.46 \pm 1.30$ & $5.16 \pm 1.45$ & $3.92 \pm 1.08$ & $3.47 \pm 1.01$ & $\mathrm{~F}_{3,122}=1.148$ & 0.0205 \\
\hline \multicolumn{7}{|c|}{ IDF } \\
\hline Total Testosterone (ng/ml) & $5.48 \pm 1.33$ & $6.69 \pm 1.58$ & $8.38 \pm 1.54$ & $2.44 \pm 0.99$ & $\mathrm{~F}_{3,122}=1.0930$ & 0.3590 \\
\hline Free Testosterone (ng/ml) & $0.33 \pm 0.00$ & $0.30 \pm 0.00$ & $0.15 \pm 0.00$ & $0.10 \pm 0.00$ & $\mathrm{~F}_{3,122}=1.2140$ & 0.3123 \\
\hline Bioavailable Testosterone (ng/ml) & $5.12 \pm 1.48$ & $6.36 \pm 1.08$ & $7.51 \pm 1.92$ & $2.57 \pm 0.72$ & $\mathrm{~F}_{3,122}=1.1550$ & 0.3342 \\
\hline SHBG (nmol/1) & $5.02 \pm 1.37$ & $4.38 \pm 1.03$ & $4.08 \pm 1.03$ & $3.22 \pm 1.05$ & $\mathrm{~F}_{3,122}=0.7433$ & 0.2853 \\
\hline \multicolumn{7}{|c|}{ WHO } \\
\hline Total Testosterone (ng/ml) & $11.49 \pm 2.61$ & $6.66 \pm 1.38$ & $5.96 \pm 1.52$ & $3.48 \pm 0.81$ & $\mathrm{~F}_{3,122}=0.4250$ & 0.7357 \\
\hline Free Testosterone (ng/ml) & $0.42 \pm 0.09$ & $0.31 \pm 0.00$ & $0.20 \pm 0.09$ & $0.10 \pm 0.04$ & $\mathrm{~F}_{3,122}=0.3386$ & 0.7974 \\
\hline Bioavailable Testosterone (ng/ml) & $10.93 \pm 2.04$ & $6.28 \pm 1.29$ & $5.85 \pm 1.47$ & $3.35 \pm 0.73$ & $\mathrm{~F}_{3,122}=0.4560$ & 0.7140 \\
\hline SHBG (nmol/l) & $5.83 \pm 1.62$ & $4.33 \pm 1.35$ & $4.01 \pm 1.86$ & $3.18 \pm 1.00$ & $\mathrm{~F}_{3,122}=0.3266$ & 0.3820 \\
\hline
\end{tabular}


When the hormonal parameters were considered as determinants with adjustment for confounders, only low levels of free and bioavailable testosterone were significant determinants of the MetS, with an aOR of 1.5 and 4.5 respectively. None of the parameters were determinants of either SD or SD/MetS (Tables 7-9).

\section{Discussion}

It is of interest the observation that participants with the MetS had lower levels of SHBG and lower levels of total, free and bioavailable testosterone in comparison to those without the MetS. Low SHBG and testosterone levels have been reported in some studies as a risk factor of the MetS [25]. Similarly hypogonadism

Table 7. Hormonal determinants of MetS in males.

\begin{tabular}{|c|c|c|c|c|}
\hline Variable & OR (CI) & Pvalue & aOR (CI) & $P$ Value \\
\hline \multicolumn{5}{|c|}{ Tot. Testosterone } \\
\hline \multicolumn{5}{|l|}{ Normal } \\
\hline Low & $4.27(0.91-19.9903)$ & 0.046 & $5.11(0.31-84.00)$ & 0.2540 \\
\hline \multicolumn{5}{|c|}{ Free Testosterone } \\
\hline \multicolumn{5}{|l|}{ Normal } \\
\hline \multirow[t]{2}{*}{ Low } & $3.17(0.91-11.06)$ & 0.0410 & $1.50(0.43-7.72)$ & 0.0312 \\
\hline & \multicolumn{3}{|c|}{ Bioavailable Testosterone } & \\
\hline \multicolumn{5}{|l|}{ Normal } \\
\hline Low & $3.90(1.06-14.31)$ & 0.0400 & $4.49(1.13-17.84)$ & 0.0330 \\
\hline \multicolumn{5}{|l|}{ SHBG } \\
\hline \multicolumn{5}{|l|}{ Normal } \\
\hline Low & $2.28(0.42-12.39)$ & 0.3410 & $2.64(0.42-16.78)$ & 0.3040 \\
\hline
\end{tabular}

Table 8. Hormonal Determinants of SD in Diabetic males.

\begin{tabular}{|c|c|c|c|c|}
\hline Variable & OR (CI) & P Value & $\mathrm{aOR}(\mathrm{CI})$ & $P$ Value \\
\hline \multicolumn{5}{|c|}{ Tot. Testosterone } \\
\hline \multicolumn{5}{|l|}{ Normal } \\
\hline Low & $0.90(0.20-4.17)$ & 0.8940 & $1.11(0.22-5.67)$ & 0.8990 \\
\hline \multicolumn{5}{|c|}{ Free Testosterone } \\
\hline \multicolumn{5}{|l|}{ Normal } \\
\hline Low & $1.30(0.35-4.78)$ & 0.6980 & $1.37(0.35-5.34)$ & 0.6520 \\
\hline \multicolumn{5}{|c|}{ Bioavailable Testosterone } \\
\hline \multicolumn{5}{|l|}{ Normal } \\
\hline Low & $1.12(0.30-4.21)$ & 0.8690 & $1.20(0.30-4.78)$ & 0.7980 \\
\hline \multicolumn{5}{|l|}{ SHBG } \\
\hline \multicolumn{5}{|l|}{ Normal } \\
\hline Low & $2.97(0.33-27.13)$ & 0.9660 & $2.35(0.25-21.83)$ & 0.4520 \\
\hline
\end{tabular}


Table 9. Hormonal determinants of SD/MetS in Diabetic males.

\begin{tabular}{|c|c|c|c|c|}
\hline Variable & OR (CI) & $P$ Value & $\mathrm{aOR}(\mathrm{CI})$ & P Value \\
\hline \multicolumn{5}{|c|}{ Tot. Testosterone } \\
\hline \multicolumn{5}{|l|}{ Normal } \\
\hline Low & $1.13(0.20-6.27)$ & 0.8900 & $0.50(0.03-7.57)$ & 0.6200 \\
\hline \multicolumn{5}{|c|}{ Free Testosterone } \\
\hline \multicolumn{5}{|l|}{ Normal } \\
\hline Low & $1.66(0.43-6.41)$ & 0.4650 & $0.96(0.16-5.89)$ & 0.9610 \\
\hline \multicolumn{5}{|c|}{ Bioavailable Testosterone } \\
\hline \multicolumn{5}{|l|}{ Normal } \\
\hline Low & $1.91(0.48-7.53)$ & 0.3550 & $1.03(0.16-6.52)$ & 0.9780 \\
\hline \multicolumn{5}{|l|}{ SHBG } \\
\hline \multicolumn{5}{|l|}{ Normal } \\
\hline Low & $1.77(0.29-10.76)$ & 0.5360 & $0.67(0.06-7.31)$ & 0.7390 \\
\hline
\end{tabular}

have been reported in some studies to be associated with the MetS [3] [22] [26] and obesity [22] [27]. Even though available evidence has pointed to decreasing testosterone levels with aging among diabetic subjects, it is possible that increasing adiposity which is a cardinal event underpinning the MetS and obesity could be inhibiting testosterone production via leptin receptors present on leydig cells or via increased aromatization of gonadal androgens to estrogens. Thus the finding of low testosterone levels in participants with obesity. This study also reports a decreased SHBG levels among participants with increased central obesity, obesity has strongly been linked to decreasing levels of gonadal androgens either due to the increased aromatization or a direct inhibition via negative feedback. Irrespective of which of these mechanisms is chiefly at play in the linkage between hypogonadism and obesity, it is without doubt the fact that increased adiposity has a linkage with decreasing SHBG levels as shown by the relationship between central obesity and SHBG levels in this study. It is also interesting that subjects with increased triglycerides levels showed a significant decrease in the levels of their total, bioavailable and free testosterone. It is established that hypertriglyceridemia is an early occurrence in the development of the MetS and thus the development of hypogonadism by participants with hypertriglyceridemia could possibly be related to the relationship of hypogonadism and triglycerides levels as demonstrated by Agledahl and his colleagues [28] who reported that men with low testosterone had increased triglycerides levels, it is therefore in agreement with the findings in this study, as participants with low testosterone levels recorded higher triglyceride levels. Free and bioavailable testosterone levels was shown in this study to be true markers of hypogonadism and determinants of the MetS. This also establishes the role of hypogonadism as a cardinal event in the dyslipidaemic derangements that eventually manifests as the MetS. It is therefore not surprising that low levels of free and bioavailable 
testosterone recorded as independent determinants of the MetS after adjustment for confounders. Participants with difficulties of sexual avoidance and sexual infrequency recorded lower and hypogonadal levels of total, bioavailable and free testosterone. This also establishes the very crucial role of testosterone and its bioactive components as very important agents in male sexual libido. The presence of hypogonadism among this study participants had a strong influence on the frequency with which they engaged in sexual activity as well as the rate at which they avoided sexual activity. This then support what has been established in other studies that hypogonadism is associated with male libido and sexual desire [29]. Since hypogonadism is strongly associated with both hypertriglyceridaemia as well as avoidance and infrequency of sexual activity, it is thus possible that hypogonadism could be the possible link between the MetS and SD. The role of hypogonadism as a possible linkage between MetS and SD could be a very important pathophysiological link that is further supported by the increased prevalence of SD and the MetS seen among hypogonadal patients. Participants with low bioavailable testosterone levels had about four and half times likelihood of developing the MetS whilst those with low levels of free testosterone had about one and half times likelihood of developing the Mets, bioavailable testosterone therefore is possibly a stronger determinant of MetS than free testosterone levels. This is not surprising as the components of testosterone that is free and that which is bound to albumin, collectively referred to as bioavailable testosterone, are readily available for biological action and better reflects the availability levels of bioactive testosterone than free testosterone alone. Thus the finding in this study that participants with central obesity recorded lower SHBG levels could explain why bioavailable testosterone levels was a better determinant of the MetS than free testosterone levels, as decreasing SHBG levels was more likely to influence bioavailable testosterone levels than free testosterone levels, thus bioavailable testosterone levels could possibly be a better marker of hypogonadism than free testosterone levels, and also that central obesity could be more associated with reductions in SHBG levels and with hypogonadism than is abdominal obesity. This finding agrees with the findings of Morris and his colleagues [30] in which total testosterone levels reflected the bioactive components better than free testosterone, with free testosterone levels reported to be useful only when total testosterone levels was on the borderline. However even though this study also found total testosterone to be a determinant of the MetS, this was found not to be an independent observation and thus the bioavailable testosterone component was a better marker of hypogonadism than the free testosterone component.

\section{Conclusion}

Testosterone and its bioactive components have a very strong association with male libido as well as the metabolic syndrome. Low levels of free and bioavailable testosterone are true determinants of the MetS in men. Bioavailable testosterone levels are a stronger determinant of the MetS than free testosterone levels. 


\section{Competing Interests}

The authors declare that they have no competing interests.

\section{Authors' Contributions}

WKBAO, NA, HA, ATB and CKGS developed the concept and designed the study. NA, WKBAO, HA, PPMD, EBAP and ATB administered the questionnaire, analysed and interpreted the data.NA, WKBAO, HA, PPMD and ATB performed all the assay procedures. WKBAO, HA, NA, PPMD, ATB and EBAPdrafted the manuscript. NA, HA, WKBAO, PPMD, CKGS and ATB revised the manuscript for intellectual content. All authors read and approved the final manuscript.

\section{Ethics Approval and Consent for Participation}

Ethical approval was obtained from the Committee on Human Research Publication and Ethics (CHRPE) of the School of Medical Science and the Komfo Anokye Teaching Hospital (KATH), Kumasi. All participants gave an informed and signed consent to partake in this study.

\section{Consent for Publication}

All participants gave an informed and signed consent for the research to be used for publication.

\section{References}

[1] Kolodny, L. (2003) Erectile Dysfunction and Vascular Disease. What Is the Connection? Postgraduate Medicine, 114, 30-40. https://doi.org/10.3810/pgm.2003.10.1504

[2] Toone, B.K., Wheeler, M., Nanjee, M., Fenwick, P. and Grant, R. (1983) Sex Hormones, Sexual Activity and Plasma Anticonvulsant Levels in Male Epileptics. Journal of Neurology, Neurosurgery, and Psychiatry, 46, 824-826. https://doi.org/10.1136/jnnp.46.9.824

[3] Amidu, N., Owiredu, W.K.B.A., Gyasi-Sarpong, C.K., Alidu, H., Antuamwine, B.B. and Sarpong, C. (2017) The Inter-Relational Effect of Metabolic Syndrome and Sexual Dysfunction on Hypogonadism in Type II Diabetic Men. International Journal of Impotence Research, in press. https://doi.org/10.1038/ijir.2017.6

[4] O'Carroll, R., Shapiro, C. and Bancroft, J. (1985) Androgens, Behaviour and Nocturnal Erection in Hypogonadal Men: The Effects of Varying the Replacement Dose. Clinical Endocrinology, 23, 527-538. https://doi.org/10.1111/j.1365-2265.1985.tb01113.x

[5] Skakkebaek, N., Bancroft, J., Davidson, D. and Warner, P. (1981) Androgen Replacement with Oral Testosterone Undecanoate in Hypogonadal Men: A Double Blind Controlled Study. Clinical Endocrinology, 14, 49-61. https://doi.org/10.1111/j.1365-2265.1981.tb00364.x

[6] Kandeel, F.R., Koussa, V.K. and Swerdloff, R.S. (2001) Male Sexual Function and Its Disorders: Physiology, Pathophysiology, Clinical Investigation, and Treatment. Endocrine Reviews, 22, 342-388. https://doi.org/10.1210/edrv.22.3.0430

[7] Davidson, J.M., Kwan, M. and Greenleaf, W.J. (1982) Hormonal Replacement and Sexuality in Men. Clinics in Endocrinology and Metabolism, 11, 599-623. 
[8] Heaton, J.P. and Morales, A. (2003) Endocrine Causes of Impotence (Nondiabetes). Urologic Clinics of North America, 30, 73-81. https://doi.org/10.1016/S0094-0143(02)00115-5

[9] Hatzimouratidis, K., Amar, E., Eardley, I., Giuliano, F., Hatzichristou, D., Montorsi, F., Vardi, Y. and Wespes, E. (2010) Guidelines on Male Sexual Dysfunction: Erectile Dysfunction and Premature Ejaculation. European Urology, 57, 804-814. https://doi.org/10.1016/j.eururo.2010.02.020

[10] Coretti, G. and Baldi, I. (2007) The Relationship between Anxiety Disorders and Sexual Dysfunction. Psychiatric Times, 24, 16-21.

[11] Mikhail, N. (2006) Does Testosterone Have a Role in Erectile Function? The American Journal of Medicine, 119, 373-382. https://doi.org/10.1016/j.amjmed.2005.07.042

[12] Braga-Basaria, M., Dobs, A.S., Muller, D.C., Carducci, M.A., John, M., Egan, J. and Basaria, S. (2006) Metabolic Syndrome in Men with Prostate Cancer Undergoing Long-Term Androgen-Deprivation Therapy. Journal of Clinical Oncology, 24, 3979-3983. https://doi.org/10.1200/JCO.2006.05.9741

[13] Wang, C., Cunningham, G., Dobs, A., Iranmanesh, A., Matsumoto, A.M., Snyder, P.J., Weber, T., Berman, N., Hull, L. and Swerdloff, R.S. (2004) Long-Term Testosterone Gel (AndroGel) Treatment Maintains Beneficial Effects on Sexual Function and Mood, Lean and Fat Mass, and Bone Mineral Density in Hypogonadal Men. The Journal of Clinical Endocrinology \& Metabolism, 89, 2085-2098. https://doi.org/10.1210/jc.2003-032006

[14] McTernan, P.G., McTernan, C.L., Chetty, R., Jenner, K., Fisher, F.M., Lauer, M.N., Crocker, J., Barnett, A.H. and Kumar, S. (2002) Increased Resistin Gene and Protein Expression in Human Abdominal Adipose Tissue. The Journal of Clinical Endocrinology \& Metabolism, 87, 2407-2410. https://doi.org/10.1210/jcem.87.5.8627

[15] Chamness, S.L., Ricker, D.D., Crone, J.K., Dembeck, C.L., Maguire, M.P., Burnett, A.L. and Chang, T.S. (1995) The Effect of Androgen on Nitric Oxide Synthase in the Male Reproductive Tract of the Rat. Fertility and Sterility, 63, 1101-1107.

[16] Zvara, P., Sioufi, R., Schipper, H., Begin, L. and Brock, G. (1995) Nitric Oxide Mediated Erectile Activity Is a Testosterone Dependent Event: A Rat Erection Model. International Journal of Impotence Research, 7, 209-219.

[17] Akishita, M., Hashimoto, M., Ohike, Y., Ogawa, S., Iijima, K., Eto, M. and Ouchi, Y. (2007) Low Testosterone Level Is an Independent Determinant of Endothelial Dysfunction in Men. Hypertension Research, 30, 1029-1034. https://doi.org/10.1291/hypres.30.1029

[18] Montalcini, T., Gorgone, G., Gazzaruso, C., Sesti, G., Perticone, F. and Pujia, A. (2007) Endogenous Testosterone and Endothelial Function in Postmenopausal Women. Coronary Artery Disease, 18, 9-13.

[19] Katznelson, L., Finkelstein, J.S., Schoenfeld, D.A., Rosenthal, D.I., Anderson, E.J. and Klibanski, A. (1996) Increase in Bone Density and Lean Body Mass during Testosterone Administration in Men with Acquired Hypogonadism. The Journal of Clinical Endocrinology \& Metabolism, 81, 4358-4365.

[20] Saad, F., Gooren, L.J., Haider, A. and Yassin, A. (2008) A Dose-Response Study of Testosterone on Sexual Dysfunction and Features of the Metabolic Syndrome Using Testosterone Gel and Parenteral Testosterone Undecanoate. Journal of Andrology, 29, 102-105. https://doi.org/10.2164/jandrol.107.002774

[21] Corona, G., Mannucci, E., Ricca, V., Lotti, F., Boddi, V., Bandini, E., Balercia, G., Forti, G. and Maggi, M. (2009) The Age-Related Decline of Testosterone Is Associated with Different Specific Symptoms and Signs in Patients with Sexual Dysfunc- 
tion. International Journal of Andrology, 32, 720-728.

https://doi.org/10.1111/j.1365-2605.2009.00952.x

[22] Wang, C., Jackson, G., Jones, T.H., Matsumoto, A.M., Nehra, A., Perelman, M.A., Swerdloff, R.S., Traish, A., Zitzmann, M. and Cunningham, G. (2011) Low Testosterone Associated with Obesity and the Metabolic Syndrome Contributes to Sexual Dysfunction and Cardiovascular Disease Risk in Men with Type 2 Diabetes. Diabetes Care, 34, 1669-1675. https://doi.org/10.2337/dc10-2339

[23] NCEP (2001) Executive Summary of The Third Report of The National Cholesterol Education Program (NCEP) Expert Panel on Detection, Evaluation, and Treatment of High Blood Cholesterol In Adults (Adult Treatment Panel III). JAMA, 285, 2486-2497. https://doi.org/10.1001/jama.285.19.2486

[24] Alberti, K.G., Zimmet, P. and Shaw, J. (2006) Metabolic Syndrome-A New WorldWide Definition. A Consensus Statement from the International Diabetes Federation. Diabetic Medicine, 23, 469-480. https://doi.org/10.1111/j.1464-5491.2006.01858.x

[25] Selvin, E., Burnett, A.L. and Platz, E.A. (2007) Prevalence and Risk Factors for Erectile Dysfunction in the US. The American Journal of Medicine, 120, 151-157. https://doi.org/10.1016/j.amjmed.2006.06.010

[26] Kaplan, S.A., Meehan, A.G. and Shah, A. (2006) The Age Related Decrease in Testosterone Is Significantly Exacerbated in Obese Men with the Metabolic Syndrome. What Are the Implications for the Relatively High Incidence of Erectile Dysfunction Observed in These Men? The Journal of Urology, 176, 1524-1528. https://doi.org/10.1016/j.juro.2006.06.003

[27] Chen, Z., Maricic, M., Nguyen, P., Ahmann, F.R., Bruhn, R. and Dalkin, B.L. (2002) Low Bone Density and High Percentage of Body Fat among Men Who Were Treated with Androgen Deprivation Therapy for Prostate Carcinoma. Cancer, 95, 2136-2144. https://doi.org/10.1002/cncr.10967

[28] Agledahl, I., Skjærpe, P.-A., Hansen, J.-B. and Svartberg, J. (2008) Low Serum Testosterone in Men Is Inversely Associated with Non-Fasting Serum Triglycerides: The Tromsø Study. Nutrition, Metabolism and Cardiovascular Diseases, 18, 256262. https://doi.org/10.1016/j.numecd.2007.01.014

[29] Hintikka, J., Niskanen, L., Koivumaa-Honkanen, H., Tolmunen, T., Honkalampi, K., Lehto, S.M. and Viinamäki, H. (2009) Original Research-Endocrinology: Hypogonadism, Decreased Sexual Desire, and Long-Term Depression in Middle-Aged Men. The Journal of Sexual Medicine, 6, 2049-2057.

https://doi.org/10.1111/j.1743-6109.2009.01299.x

[30] Morris, P.D., Malkin, C.J., Channer, K.S. and Jones, T.H. (2004) A Mathematical Comparison of Techniques to Predict Biologically Available Testosterone in a Cohort of 1072 Men. European Journal of Endocrinology, 151, 241-249.

https://doi.org/10.1530/eje.0.1510241 


\section{List of Abbreviations}

\begin{tabular}{cc}
\hline ADT & Androgen Deprivation Therapy \\
AV & Avoidance \\
BP & Blood Pressure \\
DIS & Dissatisfaction \\
ED & Erectile Dysfunction \\
GRISS-M & Golombuk-Rust Inventory for Sexual Satisfaction-Male \\
IDF & International Diabetes Federation \\
IMP & Impotence \\
INF & Infrequency \\
MetS & Metabolic syndrome \\
NCEP-ATP III & National Cholesterol Education Programme-Adult Treatment Panel III \\
NO & Nitric oxides \\
NPT & Nocturnal Penile Tumescence \\
NS & Non-sensuality \\
PE & Premature Ejaculation \\
SD & Sexual Dysfunction \\
SHBG & Sex Hormone Binding Globulin \\
\hline
\end{tabular}

Submit or recommend next manuscript to SCIRP and we will provide best service for you:

Accepting pre-submission inquiries through Email, Facebook, LinkedIn, Twitter, etc. A wide selection of journals (inclusive of 9 subjects, more than 200 journals)

Providing 24-hour high-quality service

User-friendly online submission system

Fair and swift peer-review system

Efficient typesetting and proofreading procedure

Display of the result of downloads and visits, as well as the number of cited articles Maximum dissemination of your research work

Submit your manuscript at: http://papersubmission.scirp.org/

Or contact asm@scirp.org 